\title{
Strain-Induced Tissue Growth Laws: Applications to Embryonic Cardiovascular Development
}

Sandra Rugonyi*

Oregon Health \& Science University, Biomedical Engineering, 3303 SW Bond Ave, Mail Code: CH13B, Portland, USA

\begin{abstract}
Hemodynamic conditions play an essential role in the cardiovascular system, with abnormal blood flow conditions leading to growth and remodeling of cardiovascular walls. During embryonic development, altered hemodynamic conditions lead to congenital heart disease, which affects about $1 \%$ of newborn babies in developed countries. However, the mechanisms by which hemodynamic conditions affect cardiovascular development have not been fully elucidated. In this paper, we propose a model of cardiac growth in response to hemodynamic conditions, in which growth is modulated by a combination of wall strains and wall shear stresses. This is in contrast to previous models that proposed stress-induced growth laws. Because during embryonic development blood pressure increases over time, and this increase in blood pressure produces an increase in wall stresses, stress-induced growth laws would require time-dependent parameters. While blood pressure increases during development, cardiovascular walls become stiffer and thicker, and thus we postulate that instead strains experienced by cells remain approximately the same during development. This assumption motivated our cardioavascular model of strain-induced growth in response to hemodynamic conditions, which we implemented using finite element methods. Model simulations show that the proposed model results in tissue growth that is physiologically reasonable. Further, our analyses demonstrate that mechanical coupling - that results from residual stresses originating from differential tissue growth - may play a more important role in the modulation of cardiovascular tissue growth and remodeling than currently acknowledged.
\end{abstract}

Keywords: Cardiovascular development; Tissue growth; Congenital heart disease; Hemodynamic conditions during development

\section{Introduction}

During embryonic development and beyond, hemodynamic conditions play an essential role in growth and remodeling of the cardiovascular system [1-7]. Because the heart beats, generating cyclic pulsatile flow waves, there is a dynamic interaction between cardiovascular tissue and blood flow. This interaction determines the mechanical stimuli (stresses and strains/stretches) to which cardiovascular cells are subjected $[3,4,8,9]$. Cells respond to changes in mechanical stimuli by altering their gene expression and, ultimately, their function. It is currently accepted that mechanical stimuli modulates growth (change in volume) and remodeling (change in composition) of cardiovascular tissues during development and adulthood.

To assess the influence of blood flow on cardiac development, researchers have disrupted hemodynamic conditions and thus mechanical stimuli in vivo, in otherwise normal animal models of cardiac development, [2,10-12]. Alterations of hemodynamic conditions resulted in a spectrum of heart defects [1,2,10,12], including malformations of the aortic arch, ventricular septal defects, semilunar valve anomalies, and atrioventricular anomalies - all of which resemble defects found in humans with congenital heart disease, which affects about $1 \%$ of newborn babies in the US. These studies demonstrated the importance of blood flow in cardiac development and confirmed that abnormal blood flow conditions lead to cardiac defects. Researchers have further hypothesized that blood flow dynamics play an essential role in normal cardiac development $[1,13]$, that many cardiac developmental processes do not occur in the absence of normal flow, and that these developmental processes are modulated by blood flow. In other words, cardiac development is regulated not only by preprogrammed genetic processes but also by blood flow dynamics.

In the mature cardiovascular system, growth and remodeling in response to changes in hemodynamic conditions tend to restore homeostatic (equilibrium) conditions [14-16]. Therefore, most modeling studies assume that growth and remodeling of cardiovascular tissue results from deviations of wall stresses from a 'growthequilibrium' (homeostatic) stress [5,6,14-18]. Growth and remodeling then occur in an attempt of tissues (cells within the tissues) to go back to the homeostatic stress.

During embryonic development the cardiovascular system is continuously adapting to changes in hemodynamic conditions: blood pressure, $p$, and blood volume flow rate, $Q$, continuously increase with developmental stages $[3,8,19]$ to meet the growing demand of the developing embryo. Therefore a homeostatic stress condition cannot be identified, as wall stresses continuously increase during development. If cardiac growth is modeled in response to wall stresses, growth-law parameters (e.g. the growth-equilibrium stress) must change with developmental time $[17,18,20]$. This is certainly a possibility, as cell responses do change over developmental stages. However, due to the fast pace at which $p$ and $Q$ change during embryogenesis, cell responses would need to change continuously and in concert with developmental processes.

While cardiovascular wall stresses continuously increase over developmental stages, individual cells in the developing heart and

*Corresponding author: Sandra Rugonyi, Associate Professor, Oregon Health \& Science University, Biomedical Engineering, 3303 SW Bond Ave, Mail Code: CH13B, Portland, USA, E-mail: rugonyis@ohsu.edu

Received January 28, 2013; Accepted February 21, 2013; Published February 28, 2013

Citation: Rugonyi S (2013) Strain-Induced Tissue Growth Laws: Applications to Embryonic Cardiovascular Development. J Appl Mech Eng S11: 001 doi:10.4172/2168-9873.S11-001

Copyright: (c) 2013 Rugonyi S. This is an open-access article distributed under the terms of the Creative Commons Attribution License, which permits unrestricted use, distribution, and reproduction in any medium, provided the original author and source are credited. 
blood vessels may likely experience similar deformations (strains/ stretches) over the cardiac cycle throughout development (or at least defined developmental periods). This is because as the embryo grows cardiovascular walls get thicker and tissues get stiffer. Thus strain/stretch induced growth laws might be more appropriate than stress-induced growth laws to model cardiovascular growth and remodeling in response to hemodynamic conditions during embryonic development. The assumption that growth and remodeling in response to hemodynamic conditions depends on tissue strains/stretches is consistent with mounting evidence that supports the concept of straininduced mechanotransduction mechanisms [21-24].

In this paper, we will use strain-induced growth laws, and we will explore the consequences of applying these laws to growth and remodeling of the cardiovascular system. Our objective is to determine the extent to which strain-induced growth laws can explain cardiovascular growth and remodeling phenomena observed during embryonic development. To this end, we will use a simplified, cylindrical model of a developing cardiovascular vessel. Note that, at very early stages of cardiac development, the heart has a tubular structure [25], and thus results and conclusions from our model are applicable to developing vascular vessels and the developing heart.

\section{Mathematical Model of Growth}

We used continuum mechanics equations - that enforce equilibrium of forces while tissue is growing - in a simplified model of developing cardiovascular vessels, to quantify stresses and strains in response to increases in $p$ and $Q$.

\section{Kinematic description}

To mathematically describe deformation, a deformation gradient tensor, $\mathbf{F}$, is used. $\mathbf{F}$ describes deformation between a reference, stressfree configuration, and a physiological configuration at time $t$. When tissue is growing, there are two contributions to $\mathbf{F}: 1)$ tissue growth, represented by $\mathbf{F}^{\mathrm{g}}$, and 2) tissue deformation in response to applied forces and geometrical constraints, represented by $\mathbf{F}^{\mathrm{e}}$. Because tissue is incompressible, $\operatorname{det}\left(\mathbf{F}^{e}\right)=1$, however, as tissue grows, $\operatorname{det}\left(\mathbf{F}^{\mathfrak{g}}\right)>1$ if tissue volume increases, and $\operatorname{det}\left(\mathbf{F}^{\mathbf{g}}\right)<1$ if tissue volume decreases. $\mathbf{F}$ is then expressed as the result of the two contributions $[16,17,20]$,

\section{$\mathbf{F}=\mathbf{F}^{\mathrm{e}} \mathbf{F}^{\mathrm{g}}$}

Tissue grows into a stress-free configuration, and thus $\mathbf{F}^{\mathrm{g}}$ does not generate stresses [14,16-18,20]; stresses are a function of $\mathbf{F}^{e}$.

\section{Mass production/removal}

As cardiovascular tissue grows and remodels in response to hemodynamic conditions, mass production and removal from the tissue need to be accounted. Following Humphrey et al. [5], $[15,16]$, mass production is defined by $\frac{d}{d t} \int_{v} \mid \rho d v=\int_{v} m d V$

where $V$ is tissue volume at time $t$, and $m$ is the 'density production' within the tissue. If $\mathrm{V}_{0}$ is the tissue volume in the reference configuration (at time $t=0$ ), then $\mathrm{d} V=\operatorname{det} \mathbf{F} \mathrm{dV}_{0}[5,16]$, and Eq. 2 becomes

$$
\frac{d}{d t} \int_{v_{0}} \rho \operatorname{det} \boldsymbol{F} d V_{0}=\int v_{0} m \operatorname{det} \boldsymbol{F} d v_{0}
$$

Using Eq. 3 we can now introduce the time derivative inside the integral to obtain

$$
\frac{d}{d t}(\rho \operatorname{det} \mathbf{F})=m \operatorname{det} \mathbf{F}
$$

If we further assume that $\rho$ is constant, and that tissue is incompressible (i.e. det $\mathbf{F}^{\mathbf{e}}=1$ ), using Eq. 1 and Eq.4 we get

$$
\operatorname{det} F g=\exp \left(\frac{1}{\rho} \int_{0}^{t} m d \tau\right)
$$

Constitutive growth laws can be developed by specifying the change of the density production, $m$, with time.

During cardiovascular development, $m$ depends on:

1) mechanical stimuli from hemodynamic conditions and 2) preprogrammed developmental processes-that dictate for instance cell differentiation, migration and apoptosis-and that are independent of hemodynamic conditions. Mechanical stimuli thus modulate pre-programmed developmental processes. Here, we will focus on the modulation of the density production, $m$, by mechanical stimuli generated by blood flow.

\section{Linearized equations of mass production and momentum conservation}

Our objective is to better understand the implications of strain-induced growth laws on cardiovascular development. While cardiovascular wall tissues are likely nonlinear materials, and wall motion might undergo large deformations (especially in the heart) in response to hemodynamic conditions, we will focus here on qualitative analysis of growth laws. To this end, in this study we used a linearized theory of growth, assuming that addition/removal of wall tissue is infinitesimal, the wall behaves as a linear elastic material, and changes in hemodynamic conditions result in small deformations of the cardiovascular wall. Analysis of a simplified linear theory of growth, however, allows us to get important insights into the process of growth and its effects on cardiovascular tissue. A nonlinear theory will be necessary for more accurate predictions; but growth laws and growth scenarios can be studied more effectively using a linearized approach as a first step.

When employing a small strain/small displacement formulation of motion, and in this case growth, the infinitesimal strain tensor, $\mathcal{E}$, and the infinitesimal growth strain tensor, $\varepsilon^{g}$, are employed. $\varepsilon$ is a linearization of the Green-Lagrange strain tensor, $\mathbf{E}=1 / 2(\mathbf{F T} \mathbf{F}-\mathbf{I})$, which is frequently employed in finite (nonlinear) formulations of motion. $\varepsilon^{g}$ depends on mass production/removal, and can thus be derived frm Eq. 5. If total density prduction is small, i.e. $1 / \rho \int_{0}^{t} m d \tau<<1$, then

$$
\begin{aligned}
& \exp \left(\frac{1}{\rho} \int_{0}^{t} m d \tau\right) \cong 1+\frac{1}{\rho} \int_{0}^{t} m d \tau \\
& \text { and } \operatorname{det} F^{g}-1 \cong \varepsilon_{i i}^{g}
\end{aligned}
$$

where $\varepsilon_{i i}^{g}=\operatorname{tr}\left(\varepsilon^{g}\right)$. Using Eq. 5 to Eq. 7, the relationship between mass production and the infinitesin tensor is,

$$
\varepsilon_{i i}^{g}=\frac{1}{\rho} \int_{0}^{t} m d \tau
$$

To calculate tissue deformations, we use the force equilibrium equations $[16,18]$

$$
\nabla . \rho=0 ;
$$

where $\sigma$ is the Cauchy stress tensor, $\nabla$. is the divergence operator.

Constitutive relations the stress tensor, $\boldsymbol{\sigma}$, are

$$
\rho=c\left(\varepsilon-\varepsilon^{g}\right)
$$


where $\mathbf{C}$ is the linear-elastic stress-strain (stiffness) tensor, which relates stress and strain. Like in the case of finite strain theories, compatible growth strains, which result in $\left(\varepsilon-\varepsilon^{\mathcal{S}}\right)$, do not generate stresses, and residual stresses arise from non-compatible growth strains.

\section{Cylindrical model of the developing cardiovasculature}

At very early stages of development, the embryonic heart is a tube that pumps blood through a peristaltic-like motion [3,4,11]. The tubular heart, as well as the rest of the embryonic circulation, are very sensitive to hemodynamic conditions, and thus in this paper we focused on early cardiovascular development [1]. We modeled a developing cardiovascular vessel as a thick-walled cylindrical structure of inner radius $R_{i}$ and outer radius $R_{o}$, with linear-elastic walls (see also $[19,20])$. Axial growth and deformations were neglected. This model, while simple, represents the structure of the primitive, tubular heart as well as vascular vessels. In our model, in the reference configuration (time $t=0$ ) the cylindrical vessel was assumed to be in a stress-free configuration. An internal (intramural) blood pressure, $p$, was then applied to the inner surface of the cylinder. To solve for deformations, we applied Eq. 9 in cylindrical coordinates, with boundary conditions

$$
\begin{aligned}
& \left.\sigma_{r r}\right|_{r}=R_{i}=-p \\
& \sigma_{r r \mid r}=R_{0}=o
\end{aligned}
$$

where $r$ is radial position within the cylindrical model of the developing heart and $\sigma_{\mathrm{rr}}$ are radial stresses. Tissue growth depends on tissue density production, $m$, according to Eq. 8, and in cylindrical coordinates is, $\varepsilon_{\theta \theta}^{g}+\varepsilon_{r r}^{g}=\frac{1}{\rho} \int_{0}^{t} m d \tau(12)$ an

where $\varepsilon_{\theta \theta}^{g}$ and $\varepsilon_{r r}^{g}$ are growth strains in the circumferential and radial directions, respectively.

In our model, wall shear stress, $\tau$, is calculated from an imposed blood volume flow rate, $Q$, assuming Poiseuille (fully-developed) flow through the cylindrical vessel model. Therefore,

$$
\tau=\frac{4 \mu Q}{\pi R_{i}^{3}}
$$

Where $\mu$ is the blood viscosity.

\section{Constitutive growth laws}

We modeled growth by specifying changes in tissue density production, $m$, in response to changes in hemodynamic conditions, i.e. alterations in blood pressure, $p$, and blood volume flow rate, $Q$. While changes in $p$ and $Q$ frequently occur simultaneously, they are usually considered as two separate (yet coupled) contributions to growth and remodeling. Changes in $p$ result in alterations in wall stresses and strains throughout the wall thickness, according to Eq. 9 to Eq. 11; whereas changes in $Q$ result in alterations of wall shear stress, $\tau$, at the tissue-lumen interface, Eq. 13. During development, both $p$ and $Q$ increase with developmental time.

The mechanotransduction mechanisms that lead to growth modulation due to mechanical stimuli are not well understood. Our approach to modeling growth in response to increases in $p$ and $Q$ was then partially based on observations made on the response of the mature cardiovascular system to changes in hemodynamic conditions. When studying growth and remodeling of mature blood vessels in response to changes in $p$, it is frequently assumed that circumferential stress, $\sigma_{\theta \theta}$, modulates growth in both the radial and circumferential directions (and $\varepsilon_{\theta \theta}^{g}$ and $\varepsilon_{r r}^{g}$, respectively) [14-16,18]. In those studies, axial stresses are frequently neglected, and radial stresses, $\sigma_{\text {rr }}$ are usually not considered as triggers of growth because: i) they are usually smaller than circumferential strains; ii) their value is determined from boundary conditions, Eq. 11; and iii) upon imposing a transmural $p$, radial stresses are compressive, whereas circumferential stresses are tensile, and large tensile stresses are associated with tissue rupture. Based on those studies, and since $\sigma_{\theta \theta}$ mainly depends on $\varepsilon_{\theta \theta}\left(\sigma_{\theta \theta} \cong \mathrm{E} \varepsilon_{\theta \theta}\right)$, we assumed that growth was modulated by circumferential strain, $\varepsilon_{\theta \theta}$, and that growth in the circumferential and radial directions were the same $\left(\varepsilon_{\theta \theta}{ }^{g}=\varepsilon_{r r}{ }^{\mathrm{g}}\right)$. Further, we assumed that there is a growth-equilibrium strain, $\varepsilon_{\theta \theta}{ }^{*}$, at which there is no tissue growth or remodeling in response to mechanical stimuli.

The rate at which cardiovascular tissue grows in response to mechanical stimuli, might be determined by deviations from growthequilibrium conditions or might be triggered by a threshold. Both types of response are found in nature, and thus could apply to the developing cardiovasculature. If tissue growth is modulated by deviations from growth-equilibrium conditions, and since close to equilibrium a system's response can always be linearized around the equilibrium, density production can be assumed to depend linearly on deviations from the growth-equilibrium $\varepsilon_{\theta \theta}{ }^{*}$. This assumption results in a linear strain-induced growth law,

$$
m=\beta\left[\left(\varepsilon_{\theta \theta}-\varepsilon_{\theta \theta}{ }^{g}\right)-\varepsilon_{\theta \theta}^{*}\right]
$$

where $\beta$ is a rate parameter. This growth law can lead to increase or decrease of tissue volume as well as differential, localized growth.

Cardiovascular tissue growth might have a threshold behavior, in which growth occurs only if a threshold strain, such as $\varepsilon_{\theta \theta}{ }^{*}$ is reached and exceeded. Below the threshold strain, there is no growth. A threshold behavior is observed at the molecular level, when certain molecules, stretched beyond a threshold stretch, change their configurations. In the new molecular configuration, molecular domains that were 'protected' before are now available for binding, and triggering of other reactions/processes. If cardiac growth is a threshold-induced process, the density production could be modeled using threshold straininduced growth law,

$$
m=\left\{\begin{array}{lll}
\beta^{*}\left[\left(\varepsilon_{\theta \theta}-\varepsilon_{\theta \theta}{ }^{8}\right)-\varepsilon_{\theta \theta}{ }^{*}\right] & \text { if } \quad\left(\varepsilon_{\theta \theta}-\varepsilon_{\theta \theta}{ }^{8}\right)>\varepsilon_{\theta \theta}{ }^{*} \\
& \text { if } & \left(\varepsilon_{\theta \theta}-\varepsilon_{\theta \theta}{ }^{8}\right) \leq \varepsilon_{\theta \theta}{ }^{*}
\end{array}\right.
$$

where $\beta^{*}$ is a rate parameter. This growth law can lead to increase of tissue volume and differential localized growth.

Changes in $Q$ generate changes in wall shear stresses, $\tau$, which are known to be important for growth and remodeling [26-28]. In mature arteries, it has been observed that increases in wall shear stress above a homeostatic wall shear stress, $\tau^{*}$, produce an increase in vascular radius that tends to restore homeostatic values; while a decrease in shear stress below $\tau^{*}$ tends to decrease vascular radius. Growth laws in which changes in $\tau$ affect only growth in the radial direction $\left(\varepsilon_{\mathrm{rr}}{ }^{\mathrm{g}}\right)$, have been used $[14,16,18]$. In modeling cardiac growth modulation in response to changes in $Q$, we can account for the shear strain associated with $\tau$. However, shear strain is very difficult to measure, and might not be involved in all mechanotransduction mechanisms. For example, it has been shown that endothelial cells have cilia that are believed to transduce shear stresses into cell signaling [29-31]. The motion of these cilia in response to shear stress is not associated with the internal shear strain of the cell, and yet can transduce shear stress. Thus, we modeled the effects of $Q$ on tissue growth by assuming that density production, $m$, depends on $\tau$ and that there is a growth-equilibrium wall stress, $\tau^{*}$, such that

$$
m=\alpha\left(\tau-\tau^{*}\right)
$$


where $\alpha$ is a rate parameter. Since in our cylindrical cardiovascular vessel model wall shear stresses are uniform, and we will only consider cases in which $Q$ increases (and thus wall shear stress increases beyond the equilibrium $\tau^{*}$ ), considering only a linearized growth law in response to deviations from wall shear stress is enough for our study.

During growth and remodeling in vivo, both changes in $p$ and $Q$ occur together. To account for the combined effect of wall shear stress and tissue strains on growth, we combine Eq. 14 or Eq. 15 with Eq. 16, by simply adding the contributions of wall strain and wall shear stress.

\section{Finite element implementation of growth models}

We implemented the growth laws using a finite element formulation. Because tissue is incompressible, to properly solve for the deformation and growth of the cylindrical model considered, mixed $9 / 3$ elements (that satisfy the inf-sup condition) were used in a displacement/pressure ( $\mathrm{u} / \mathrm{p})$ finite element formulation [32]. Mesh independence was achieved in simulations.

\section{Results}

Non-dimensional parameters were employed in simulations of growth using our cylindrical cardiovascular vessel model. As this study is focused on analyzing consequences of the growth laws proposed (in terms of further growth and generation of residual stresses/strains), and since we are using a linearized (infinitesimal) model of deformation and growth, the actual values of the parameters are not important. We did not obtain parameters that closely represent cardiovascular mechanics, but rather main, qualitative features of cardiovascular growth were reproduced. For all the cases considered, we assumed a tissue elasticity modulus of $E=10^{6}$, and Poisson's ratio of $v=0.49$ (almost incompressible tissue). Initially ( $t=0), R i=0.5$ and $R o=1$, and thus $h=0.5$ (all in non-dimensional units), and the cylinder was assumed to be in a stress-free configuration. Then a step increase in pressure, $\Delta p=1$, and a step increase in shear stress, $\Delta \tau=2.16357 \cdot 10^{-4}$, were imposed. Growthequilibrium parameters used were $\varepsilon_{\theta \theta}{ }^{*}=1.08147 \cdot 10^{-6}$ and $\tau^{*}=10.1857$; and growth rate parameters were $\alpha / \rho=0.005, \beta / \rho=0.1$. Growth rate parameters were chosen so that growth in response to $p$ and $Q$ were about the same order of magnitude, and thus both mechanisms of growth could effectively 'compete' with each other. Growthequilibrium parameters, $\varepsilon_{\theta \theta}{ }^{*}$ and $\tau^{*}$, were chosen so that equilibrium conditions could be achieved within our assumptions.

\section{Strain-induced growth in response to step pressure}

To study the effect of the linear strain-induced growth law, Eq. 14, we applied a step internal pressure, $p$, at time $t=0$, and kept $p$ constant afterwards. At time $t=1$, the cylinder deformed in response to the applied $p$, but has not grown in response to the deformation. In our study, growth starts at time $t>1$. The initial application of $p$ produced: i) An increase in the internal and external cylinder radii, and, since the cylinder is incompressible, a decrease in the cylinder thickness (see Figure 1A, initial response); and ii) A radial distribution of circumferential strain (see Figure $1 \mathrm{~B}$, time $t=1$ ). Thus, at $t=1$, the inner 'layers' of the cylinder (up to $\mathrm{r} \sim 0.7$ ), have $\varepsilon_{\theta \theta}>\varepsilon_{\theta \theta}{ }^{*}$, while the outer 'layers' ( $r>0.7$ ) have $\varepsilon_{\theta \theta}<\varepsilon_{\theta \theta}{ }^{*}$. To reach growth-equilibrium (Eq. 14), tissue volume increased in the inner layers and decreased in the outer layers. For the case considered, Eq. 14 produced a global decrease in the volume of the cylinder, which manifested as a decrease in the thickness of the cylinder (Figure 1A). Growth-equilibrium, eharacterized by constant circumferential strains, $\left(\varepsilon_{\theta \theta}-\varepsilon_{\theta \theta}\right)=\varepsilon_{\theta \theta}$ was achieved (Figure 1B).
If growth in response to the increase in $p$ follows a threshold straininduced growth law, Eq. 15, a global decrease in tissue volume is no longer possible. Initially, only the volume of the inner layers of the cylinder increased (Figure 2B). Eventually, however, due to mechanical coupling, the volume increase of the inner cylindrical layers generated an increase in the circumferential strains of outer layers, which in turn produced an increase in volume of the outer layers as well (Figure 2B). An increase in $p$, thus results in increased thickness of the cylinder (Figure 2A).

\section{Shear-induced growth in response to step wall shear stress}

Next, we studied the effect of the shear-stress-induced growth law, Eq. 16. To this end, we applied a step increase in volume flow rate, $Q$, at time $t=0$, and kept $Q$ constant afterwards. At time $t=1, \tau$ increased in response to the increased in $Q$, but the cylinder has not grown in response to this increase. Growth starts at time $t>1$ in our study, and ends when growth-equilibrium has been achieved. We found that, in response to an increase in $\tau$, the cylinder internal and external radii increased, while the cylinder thickness showed a slight increase (Figure 3A). Growth-equilibrium was achieved by increasing internal radii and thus reducing wall shear stress to equilibrium, $\tau^{*}$. The increase in internal radius, and thus the reduction in wall shear stress, was at the
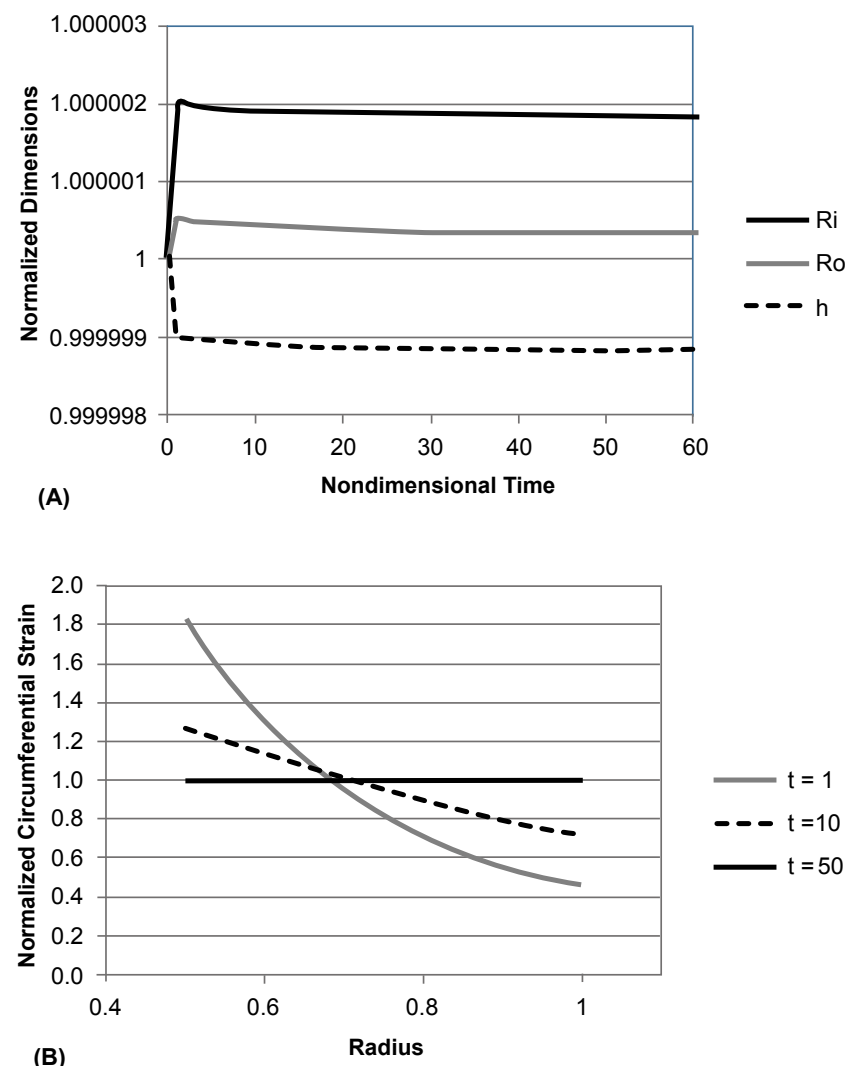

Figure 1: Growth of the cylindrical heart model after applying a step increase in intramural pressure, and assuming a linear strain-induced growth law, Eq 14. The nondimensional time $t=1$ shows the response of the model to the applied load; growth starts at $t>1$. (A) Heart dimensions over time normalized to the dimensions in the reference configuration $(t=0) ; \mathrm{Ri}$ internal radius, $\mathrm{Ro}$ outer radius, $h$ wall thickness. (B) Circumferential strains, $\varepsilon_{\theta \theta}-\varepsilon_{\theta \theta}{ }^{g}$, through the cardiac wall, normalized to the growth-equilibrium strain, $\varepsilon_{\theta \theta^{*}}$ 

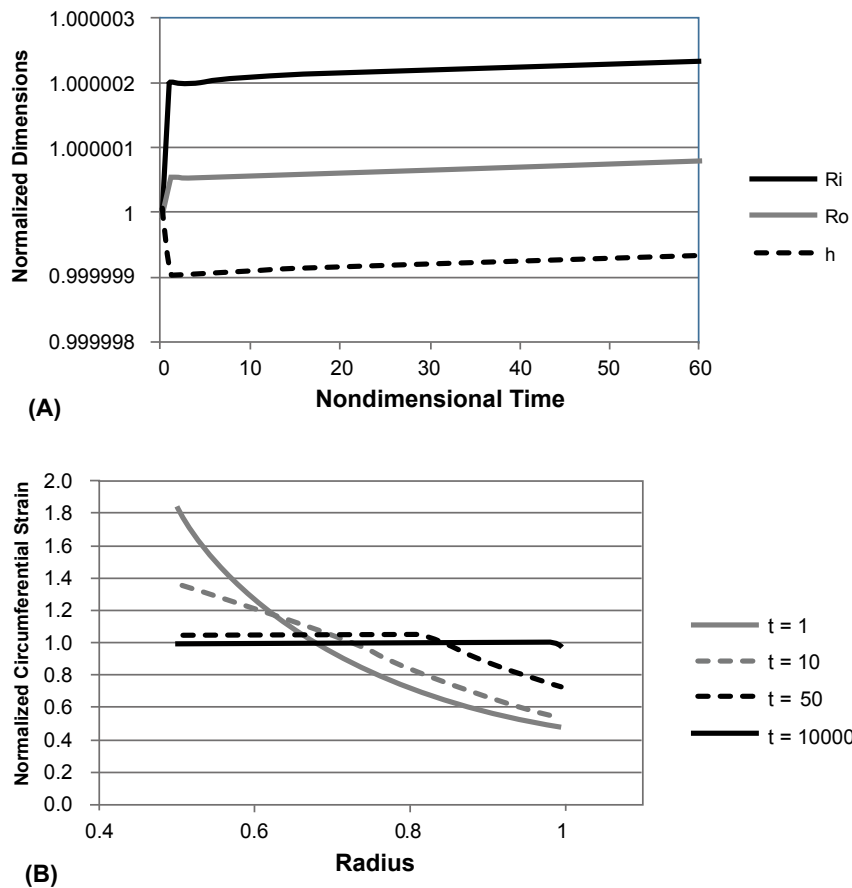

Figure 2: Growth of the cylindrical heart model after applying a step increase in intramural pressure, and assuming a threshold strain-induced growth law, Eq. 15. The nondimensional time $t=1$ shows the response of the model to the applied load; growth starts at $t>1$. (A) Heart dimensions over time normalized to the dimensions in the reference configuration $(t=0)$; $R i$ internal radius, Ro outer radius, $h$ wall thickness. (B) Circumferential strains, $\varepsilon_{\theta \theta}-\varepsilon_{\theta \theta}{ }^{g}$, through the cardiac wall, normalized to the growth-equilibrium strain, $\varepsilon_{\theta \theta}$.

expense of an increase in residual circumferential strains (Figure 3B) and thus an increase in circumferential stresses resulted.

\section{Combination of shear-stress induced and linear strain- induced growth law}

During development both blood pressure $p$ and blood volume flow rate $Q$ increase to satisfy the demands of the growing embryo. A growth law that combines the effect of both strain-induced and shearstress induced growth is therefore more appropriate. We first assumed that the growth law was given by a combination of Eq. 14 and Eq. 16. We applied a step increase in both $p$ and $Q$ at time $t=0$, and kept both $p$ and $Q$ constant afterwards. Like in previous cases considered, at time $t=1$, the cylinder deformed in response to the applied $p$, and $\tau$ increased in response to $Q$, but the cylinder has not grown: growth starts at time $t>1$, and continues until growth-equilibrium is achieved, that is until $\tau$ and circumferential strains reach their growth- equilibrium values, $\tau^{*}$ and $\varepsilon_{\theta \theta}^{*}$, respectively. We found that the cylinder internal and external radii increase, mainly in response to the change in $Q$ and thus $\tau$ (Figure $4 \mathrm{~A}$ ). The increase in radii initially produced large increases in circumferential strains, which were then decreased by strain-induced growth (Figure 4B). Note however, that the wall thickness decreased (Figure $4 \mathrm{~A})$, this is because, like in the case of purely linear straininduced growth (Figure 1), the strain-induced growth law employed, Eq. 14, resulted in a global decrease of the cylinder volume.

\section{Combination of shear-stress induced and threshold strain- induced growth law}

We next considered a combination of a threshold strain-induced growth, Eq. 15, and shear-stress-induce growth, Eq. 16. We found that in response to the increase in $p$ and $Q$, both internal and external radii increased, and the cylinder thickness also increased (Figure 5A). Initially, like in the case in which only a threshold strain-induced law, Eq. 15, was considered, the increase in $p$ produced increases in tissue volume in the inner cylinder layers. The increase in radii due to the shear-stress-induced part of the growth law, Eq. 16, exacerbated deviations of circumferential strains from equilibrium values (Figure 5B), which generated further growth of the inner layers. Later, however, due to mechanical coupling, the volume increase of the inner layers generated an increase in the strains (and stresses) of the outer layer (Figure 5B). Applying a combination of shear-induced and threshold strain-induced growth laws resulted in a more uniform increase in the volume of the cylinder (Figure 5), and both an increase in internal radius (typical response expected from an increase in wall shear stress) and an increase in wall thickness (response expected from an increase in blood pressure).

\section{Discussion}

The laws that govern the growth and remodeling of the cardiovascular system in response to hemodynamic conditions during development (and beyond) are not well understood. Growth and remodeling during development are certainly complex processes that likely include several biological mechanisms that, in turn, affect distribution of mechanical stimuli in cardiovascular walls. Mathematical modeling and computational simulation of growth, thus, can aid in the identification of proper growth laws and biological mechanisms that drive growth and remodeling.
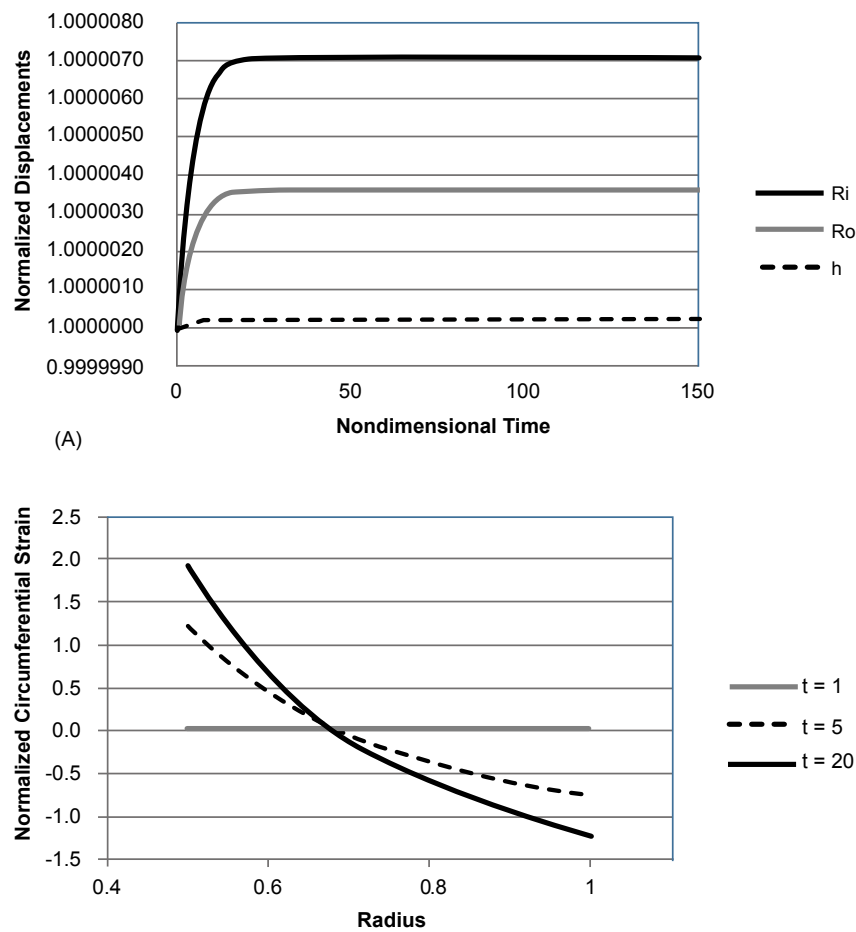

Figure 3: Growth of the cylindrical heart model after applying a step increase in blood volume flow rate, and assuming a linear shear-stress induced growth law, Eq. 16. The nondimensional time $t=1$ shows the response of the model to the applied flow; growth starts at $t>1$. (A) Heart dimensions over time normalized to the dimensions in the reference configuration $(t=0)$; Ri internal radius, Ro outer radius, $h$ wall thickness. (B) Circumferential strains, $\varepsilon_{\theta \theta}-\varepsilon_{\theta \theta}{ }^{g}$, through the cardiac wall, normalized to the growth-equilibrium strain, $\varepsilon_{\theta \theta}$. 

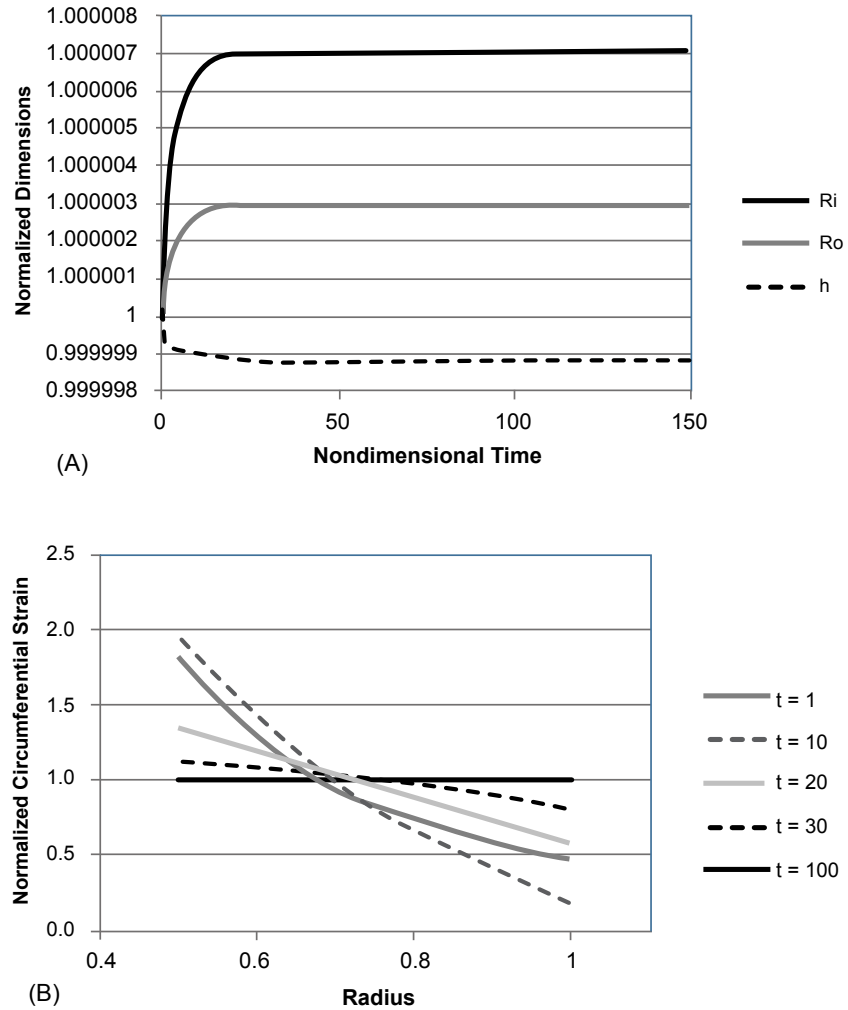

Figure 4: Growth of the cylindrical heart model after applying a step increase in intramural pressure and a step increase in blood volume flow rate, and assuming a linear strain-induced growth law combined with a linear shear-stress induced growth law, Eq. 14 and Eq. 16, respectively. The nondimensional time $t=1$ shows the response of the model to the applied load; growth starts at $\mathrm{t}>1$. (A) Heart dimensions over time normalized to the dimensions in the reference configuration $(t=0) ; R$ internal radius, Ro outer radius, $h$ wall thickness. (B) Circumferential strains, $\varepsilon_{\theta \theta}-\varepsilon_{\theta \theta}{ }^{g}$, through the cardiac wall, normalized to the growth-equilibrium strain, $\varepsilon_{\theta \theta}{ }^{*}$.

During cardiovascular development, blood pressure and blood volume flow rate increase continuously to meet the demands of the growing embryo. Thus, proper growth laws must describe continuous growth and remodeling in response to continuous changes in mechanical stimuli. Because wall stresses increase during development, while wall strains might not significantly change-as the wall becomes stiffer-strain-induced growth laws might be appropriate to describe cardiovascular growth during development.

\section{Growth-equilibrium conditions}

The growth-equilibrium strain, $\varepsilon_{\theta \theta}{ }^{*}$, and growth-equilibrium wall shear stress, $\tau^{*}$, must be such that equilibrium conditions (no growth if $p$ and $Q$ remain constant) are achievable. When using the linear strain-induced growth law, Eq. 14, if $\varepsilon_{\theta \theta}{ }^{*}$ cannot be achieved under equilibrium conditions, then the tissue volume will either decrease or increase, without stopping, even when $p$ remains constant after a step increase. When using a threshold strain-induced growth law, Eq. 15, if $\varepsilon_{\theta \theta}{ }^{*}$ is larger than strains obtained under equilibrium conditions for the given imposed $p, \varepsilon_{\theta \theta}{ }^{\text {eq }}$, then the tissue volume will not change; whereas if $\varepsilon_{\theta \theta}{ }^{*}$ is smaller than $\varepsilon_{\theta \theta}{ }^{\text {eq }}$ the tissue volume will increase without bounds (in an infinitesimal strain model). Likewise, if $\tau^{*}$ is too small, a nonphysiological, large increase in internal radius will result, whereas if $\tau^{*}$ is too large, a large lumen constriction/closure will result. Selection of a proper $\varepsilon_{\theta \theta}{ }^{*}$ and $\tau^{*}$ is an important consideration when formulating growth-laws.

The following approximations can be used to estimate equilibrium conditions,

$$
\rho_{\theta \theta}{ }^{h} \cong p R_{i}
$$

Or, if the wall behavior is approximated with that of a linear elastic $\operatorname{material}_{\varepsilon q} q \frac{p R_{i}}{E h}$

Further, Eq. 13 can be used to estimate the growth-equilibrium wall shear stress, given $Q$ and the vascular radius.

Proper selection of $\varepsilon_{\theta \theta}{ }^{*}$ is more restrictive in a linear-elastic formulation, assuming small strains and small displacements, that in the general non-linear case. During development, the cardiovascular walls become stiffer (thus the 'effective' elasticity modulus $E$ within a nonlinear formulation increases as tissue grows); further, $R i$ and $h$ also increase significantly. A continuous increase in $p$, together with a continuous increase in $R i, E$ and $h$ can lead to a constant $\varepsilon_{\theta \theta}{ }^{\text {eq }}$ (see Eq. 18). Achievement of a constant equilibrium strain, $\varepsilon_{\theta \theta}{ }^{*}=\varepsilon_{\theta \theta}{ }^{\text {eq }}$, throughout development is thus a reasonable assumption.

In the developing cardiovascular system, $Q$ increases over time.
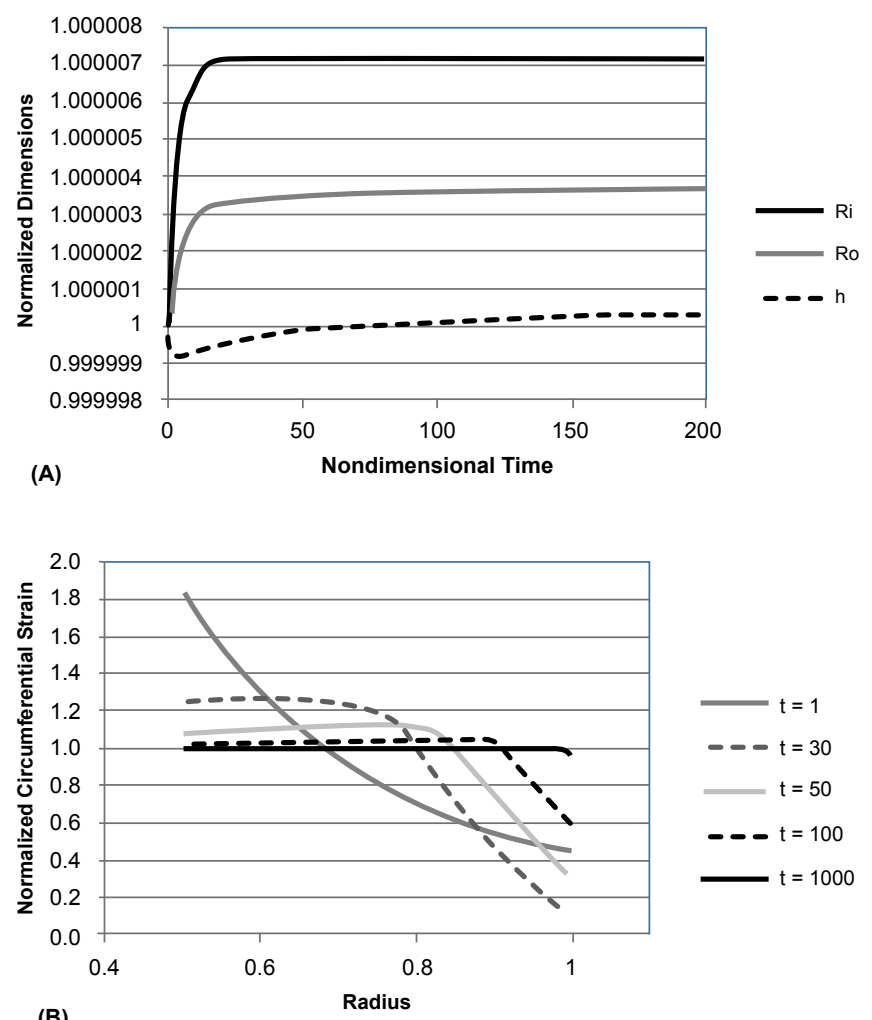

Figure 5: Growth of the cylindrical heart model after applying a step increase in intramural pressure and a step increase in blood volume flow rate, and assuming a threshold strain-induced growth law combined with a linear shear-stress-induced growth law, Eq. 15 and Eq. 16, respectively. The nondimensional time $t=1$ shows the response of the model to the applied load; growth starts at $t>1$. (A) Heart dimensions over time normalized to the dimensions in the reference configuration $(t=0)$; $R_{i}$ internal radius, Ro outer radius, $h$ wall thickness. (B) Circumferential strains, $\varepsilon_{\theta \theta}-\varepsilon_{\theta \theta}{ }^{g}$, through the cardiac wall, normalized to the growth-equilibrium strain, $\varepsilon_{\theta \theta}{ }^{*}$. 
It is however not certain whether $\tau$ also increases with development. Some studies show that $\tau$ might increase [33], but other studies show quantifications of $\tau$ during development that are not lower than typical values found in the mature cardiovascular system [34]. More studies are needed to elucidate this issue. Our assumption of a constant growth-equilibrium $\tau^{*}$ is certainly a possibility.

\section{Generation of residual stress/strain during development}

As tissue grows, residual stresses and strains arise because the 'new' tissue has to be accommodated within the vessel, and geometrical restrictions exist. This is analogous to the generation of thermal residual stresses in structural materials. Residual stresses also arise due to differential growth, which happens when tissue does not grow homogeneously, and tissue volume changes by a different amount in different locations (localized growth). Growth in response to wall stresses and/or strains generate differential growth, since, after a change in hemodynamic conditions (e.g. an increase in $p$ ), distributions of stresses and strains are not uniform. Thus, it is not surprising that the constitutive growth-laws studied here, Eq. 14 to Eq. 16 and combinations of them, generated residual stresses/strains.

It is well known that cardiovascular vessels are under residual stresses/strains (e.g. [6,5]). Transversal cuts of blood vessels produce a reduction in vessel length, which implies that in vivo the vessel is under tension, and longitudinal cuts result in an opening angle, which reveals the existence of circumferential stresses in vivo. Nowadays, it is well accepted that in a cylindrical vessel, circumferential stresses are approximately uniform throughout the thickness $[5,18]$. This distribution of stresses is believed to originate in the process of developmental growth and remodeling, which is certainly captured by our simplified models.

In this study, we showed that a uniform distribution of circumferential strains, which leads to an approximately uniform distribution of circumferential stresses, can be achieved if growth of the developing heart is modulated by circumferential strains. Using straininduced growth laws, Eq. 14 and Eq. 15, alone or in combination with wall shear stress growth laws, Eq. 16, uniform circumferential strains were achieved (Figures 1, 2, 4 and 5). However, if wall shear stress growth laws, Eq. 16, are used alone, resulting circumferential strains (and stresses) are not uniform. These results suggest that using growth laws that only account for the effect of wall shear stresses on tissue growth (more specifically radial growth) might not be physiologically relevant. However, the proposed strain-induced growth laws, alone or in combination with wall shear stress growth laws, can reproduce the uniform distribution of circumferential strains/stresses in the cardiovascular system.

\section{Growth in response to an increase in intramural pressure}

In the mature cardiovascular system, an increase in intramural pressure results in thicker cardiovascular walls. A thicker wall in turn, contributes to an increase in the global stiffness of the wall, which can then withstand higher pressures. The threshold strain-induced growth law, Eq. 16, reproduced the increase in wall thickness expected after an increase in $p$ (Figure 2), thus providing a physiologically relevant response to an increase in $p$.

The linear strain-induced growth model did not seem to reproduce the expected increase in wall thickness in response to an increase in $p$ (Figure 2). This, however, is because of the 'artificial' initial conditions chosen: at time $t=0$ the cylindrical heart was in a stressfree configuration, and then a pressure, $p$, was applied. As expected, application of $p$ strained the walls of the cylindrical vessel. To attain equilibrium, the growth-equilibrium strain, $\varepsilon_{\theta \theta}{ }^{*}$ was chosen to be above 0 . As a consequence of this choice, part of the wall had strains that were above $\varepsilon_{\theta \theta}{ }^{*}$, which then grew and increased their volume, whereas the other part of the wall had strains below $\varepsilon_{\theta \theta}{ }^{*}$, in which volume decreased. This resulted in an overall decrease of wall thickness. If choosing a more appropriate initial condition, such as having a cylindrical vessel that is strained at $t=0$ with circumferential strains equal to $\varepsilon_{\theta \theta}{ }^{*}$, an increase in $p$ will result in an overall increase in wall thickness. This is because the increase in $p$ will strain the cylindrical vessel beyond $\varepsilon_{\theta \theta}{ }^{*}$ everywhere (the same way it strained the stress-free vessel beyond 0 everywhere). Because our models are linear, this can be inferred from our results without further numerical testing. Thus volume will increase in all parts of the wall. Therefore, given the appropriate initial conditions, Eq. 15, provides a physiologically relevant response to an increase in $p$.

\section{Growth in response to an increase in blood volume flow rate}

An increase in the blood volume flow rate $Q$ that circulates through a vascular vessel increases the wall shear stress, $\tau$, on the vessel walls, Eq. 13. In the mature cardiovascular system, an increase in $\tau$ results in an increase in the diameter of the blood vessel. This increase in diameter decreases $\tau$ and restores homeostatic conditions. Wall shear stress is sensed by the endothelial layer, which is in direct contact with blood flow, and is composed of a monolayer of endothelial cells, e.g. $[26,27]$. If only the endothelial layer were to respond to changes in wall shear stress, then changes in vessel diameter will not be possible (as the endothelial-cell layer would not be strong enough to push the rest of the cardiovascular walls outwards). Cross-talk mechanisms between the endothelial layer and the other layers of the cardiovascular walls 'propagate' the effect of wall shear stress to the whole wall $[5,6,14,16]$. This is expressed mathematically by Eq. 16, which establishes that in response to an increase in $\tau$, the whole cardiovascular wall expands in the radial direction-thus all layers in a cylindrical representation of a vessel wall grow equally in response to the wall shear stress.

During development, cardiovascular vessel walls are composed by an inner endothelial layer, a monolayer of endothelial cells that directly senses wall shear stress; an outer myocardium (heart) or smoothmuscle (vessel) cell layer, as well as extra-cellular matrix $[25,35,36]$. The endothelial and myocardial/smooth-muscle cells secrete extra cellular matrix components that contribute to the formation and growth of cardiovascular tissue, presumably (at least in part) in response to mechanical stimuli $[37,38]$. Current research suggests that during growth of the cardiovascular system, endothelial and myocardial/ smooth-muscle cells act together to increase the vessel dimensions in response to shear stress (possibly with different rates), while the extracellular matrix remodels its cross-linked network to accommodate the growth of tissue [19]. Therefore, Eq. 16, provides a physiologically relevant response to changes in wall shear stress in the developing cardiovasculature.

\section{Combined growth due to blood pressure and blood volume flow rate}

Separately, Eq. 14 or Eq. 15 and Eq. 16 uncouple the growth response induced by changes in $p$ and $Q$. This uncoupling can have nonphysiological consequences. For instance, in response to an increase in $p$, the wall thickness might increase at the expense of a reduction in the internal tube radius (Figure 2), which then generates an increase in $\tau$. In response to an increase in $\tau$, the internal tube radius increases, at the expense of large increases in residual circumferential stresses/ strains (Figure 3). To avoid this physiologically unlike responses, a 
combination of both equations, Eq. 14 or Eq. 15 and Eq. 16, which will then consider responses to both deviations from circumferential strains and wall shear stresses, is needed. This combination of equations to compute growth appears to be a good approximation to growth and remodeling in response to hemodynamic conditions (Figure 4 and 5).

Combinations of shear-stress and strain induced growth laws could then be used to predict growth of the developing heart and vasculature in response to a continuous increase in $p$ and $Q$, which resemble conditions during embryonic development. To this end, a nonlinear theory of growth, which includes finite deformation and growth, would be necessary to account for the large changes in cardiovascular wall volume that occur during development, as well as developmental changes in tissue composition (not considered in our simplified model). As cardiovascular tissue volume increases, and cardiovascular walls become stiffer, it is feasible that cardiovascular growth in response to hemodynamic conditions is driven by small (bounded) deviations from growth equilibrium, as the developing cardiovasculature adapts to continuous changes in blood flow dynamics.

\section{Mechanical effects of growing tissue}

Our results suggest that mechanical coupling affects cardiovascular growth in response to hemodynamic conditions. In the examples considered, tissue growth is aided by mechanical coupling within the continuum model of the tubular vessel. For instance, when the threshold strain-induced growth law, Eq. 15, is used to compute tissue volume changes in response to a step increase in $p$ (Figure 2), a priori it seems that only the inner part of the tubular wall, in which circumferential strains are larger than the growth-equilibrium circumferential strain, $\varepsilon_{\theta \theta}{ }^{*}$,would increase in volume. However, as the inner portion of the tubular vessel grows, strain in the outer portion of the heart increase, and, in turn, the outer part of the vessel wall also grows (increase in volume) until the entire wall thickness has the same circumferential strain (Figure 2). This is also the case when a combination of responses to blood pressure and wall shear stresses, Eq. 15 and Eq. 16, is considered (Figure 5). Although not as evident, mechanical coupling also influences strains in the linear strain-induced growth law, Eq. 14, and when a combination of linear strain-induced and wall shear-stress-induced growth laws is considered. Because mechanical coupling affects distributions of stresses and strains in the walls of the developing cardiovascular system, our results suggest that mechanical coupling is an important component of growth in response to hemodynamic conditions. Mechanical coupling, however, is very difficult to determine using only experimental results, and therefore mathematical models of growth may be needed in identifying growth mechanisms during cardiovascular development.

\section{Conclusion}

In this paper, we considered growth laws that could be applied to cardiovascular growth during development, when blood pressure and blood volume flow rate increase continuously with developmental time. We proposed that strain-induced growth laws combined with wall shear stress growth laws, which account for changes in pressure and blood volume flow rate, respectively, are feasible in the modeling of developmental cardiovascular growth. Further, we showed that the proposed laws can reproduce physiological behaviors that are expected from growth and remodeling in response to hemodynamic conditions.

Mathematical modeling and simulation of cardiovascular growth revealed an interesting interaction between tissue growth and how growth affect tissue stresses/strains and could therefore promote further growth. Our results showed the importance of mechanical coupling in strain-induced (or stress-induced) growth. To fully understand the consequences of growth in terms of mechanical coupling and generation of residual strains/stresses, mathematical modeling is needed. However, to properly model growth and remodeling, experimental data is required. Thus, a combination of experimental and theoretical approaches is needed to fully understand how cardiac and vascular growth and remodeling occur in response to mechanical stimuli during developmental stages and beyond.

\section{Acknowledgements}

This work has been supported in part by grant NIH R01 HL094570. The content is solely the responsibility of the authors and does not necessarily represent the official views of grant giving bodies.

\section{References}

1. Hove JR, Koster RW, Forouhar AS, Acevedo-Bolton G, Fraser SE, et al. (2003) Intracardiac fluid forces are an essential epigenetic factor for embryonic cardiogenesis. Nature 421: 172-177.

2. Sedmera D, Pexieder T, Rychterova V, Hu N, Clark EB (1999) Remodeling of chick embryonic ventricular myoarchitecture under experimentally changed loading conditions. Anat Rec 254: 238-252.

3. Clark EB (1984) Functional aspects of cardiac development.

4. Keller BB (1998) Embryonic cardiovascular function, coupling and maturation: a species view 65-87.

5. Humphrey JD (2002) Cardiovascular solid mechanics: cells, tissues, and organs. Springer, USA

6. Fung YC (1993) Biomechanics: Mechanical Properties of Living Tissues Springer, USA.

7. Hierck BP, Van der Heiden K, Poelma C, Westerweel J, Poelmann RE (2008) Fluid shear stress and inner curvature remodeling of the embryonic heart Choosing the right lane. Sci World J 8: 212-222.

8. Hu N, Clark EB (1989) Hemodynamics of the stage 12 to stage 29 chick embryo. Circ Res 65: 1665-1670.

9. Damon BJ, Remond MC, Bigelow MR, Trusk MR, Xie W, et al. (2009) Patterns of muscular strain in the embryonic heart wall. Dev Dyn 238: 1535-1546.

10. Clark EB, Rosenquist GC (1978) Spectrum of cardiovascular anomalies following cardiac loop constriction in the chick embryo. Birth Defects Orig Artic Ser $14: 431-442$

11. Lucitti JL, Tobita K, Keller BB (2005) Arterial hemodynamics and mechanica properties after circulatory intervention in the chick embryo. J Exp Bio 208: 1877-1885.

12. Hogers B, DeRuiter M, Gittenberger-de Groot A, Poelmann RE (1999) Extraembryonic venous obstructions lead to cardiovascular malformations and can be embryolethal. Cardiovasc Res 41: 87-99.

13. Pexieder $T$ (1975) Cell death in the morphogenesis and teratogenesis of the heart. Adv Anat Embryol Cell Biol 51: 3-99

14. Taber LA, Humphrey JD (2001) Stress-modulated growth, residual stress, and vascular heterogeneity. J Biomech Eng 123: 528-535.

15. Valentin A, Humphrey JD (2009) Evaluation of fundamental hypotheses underlying constrained mixture models of arterial growth and remodeling Philos Transact A Math Phys Eng Sci 367: 3585-3606.

16. Humphrey JD, Rajagopal KR (2003) A constrained mixture model for arteria adaptations to a sustained step change in blood flow. Biomech Mode Mechanobio 2: 109-126.

17. Rodriguez EK, Hoger A, McCulloch AD (1994) Stress-dependent finite growth in soft elastic tissues. J Biomech 27: 455-467.

18. Taber LA (2009) Towards a unified theory for morphomechanics. Philos Trans Royal Soc A 367: 3555-3583.

19. Taber LA, Perucchio R (2000) Modeling heart development. Journal of elasticity and the physical science of solids $61: 165-197$. 
Citation: Rugonyi S (2013) Strain-Induced Tissue Growth Laws: Applications to Embryonic Cardiovascular Development. J Appl Mech Eng S11: 001 doi:10.4172/2168-9873.S11-001

Page 9 of 7

20. Lin I, Taber L (1995) A Model For Stress-Induced Growth In The Developing Heart. J Biomech Eng 117: 343-349.

21. Humphrey JD (2001) Stress, strain, and mechanotransduction in cells. J Biomech Eng 123: 638-641.

22. Wu Z, Wong K, Glogauer M, Ellen RP, McCulloch CA (1999) Regulation of stretch-activated intracellular calcium transients by actin filaments. Biochem Biophys Res Commun 261: 419-425.

23. Helmke BP, Rosen AB, Davies PF (2003) Mapping mechanical strain of an endogenous cytoskeletal network in living endothelial cells. Biophys J 84 2691-2699.

24. Bhole AP, Flynn BP, Liles M, Saeidi N, Dimarzio CA, et al. (2009) Mechanical strain enhances survivability of collagen micronetworks in the presence of collagenase: implications for load-bearing matrix growth and stability. Philos Transact A Math phys Eng Sci 367: 3339-3362.

25. Martinsen BJ (2005) Reference guide to the stages of chick heart embryology. Dev Dyn 233: 1217-1237.

26. Davies PF (2009) Hemodynamic shear stress and the endothelium in cardiovascular pathophysiology. Nat Clin Pract Cardiovasc Med 6: 16-26.

27. Davies PF (1995) Flow-mediated endothelial mechanotransduction. Physiol Rev 75: 519-560.

28. Poelmann RE, Gittenberger-de Groot AC, Hierck BP (2008) The development of the heart and microcirculation: role of shear stress. Med Bio Eng Comput 46: $479-484$.

29. Abou Alaiwi WA, Lo ST, Nauli SM (2009) Primary cilia: highly sophysticated biological sensors. Sensors 9: 7003-7020.
30. Groenendijk BC, Van der Heiden K, Hierck BP, Poelmann RE (2007) The role of shear stress on ET-1, KLF2, and NOS-3 expression in the developing cardiovascular system of chicken embryos in a venous ligation model. Physiology (Bethesda) 22: 380-389.

31. Van der Heiden K, Groenendijk BC, Hierck BP, Hogers B, Koerten HK, et al (2006) Monocilia on chicken embryonic endocardium in low shear stress areas. Dev Dyn 235: 19-28.

32. Bathe KJ (1996) Finite Element Procedures: Prentice-Hall.

33. Taber LA (1998) An optimization principle for vascular radius including the effects of smooth muscle tone. Biophy J 74: 109-114.

34. Poelma C, Van der Heiden K, Hierck BP, Poelmann RE, Westerweel J (2009) Measurements of the wall shear stress distribution in the outflow tract of an embryonic chicken heart. J R Soc 7: 91-103.

35. Hurle JM, Icardo JM, Ojeda JL (1980) Compositional and structura heterogenicity of the cardiac jelly of the chick embryo tubular heart: a TEM SEM and histochemical study. J Emb Exp Mor 56: 211-223.

36. Manner J (2000) Cardiac looping in the chick embryo: a morphological review with special reference to terminological and biomechanical aspects of the looping process. Anat Rec 259: 248-262.

37. Armstrong EJ, Bischoff J (2004) Heart valve development: endothelial cell signaling and differentiation. Cir Res 95: 459-470.

38. Nakashima Y, Yamagishi T, Hokari S, Nakamura H (2000) Mechanisms involved in valvuloseptal endocardial cushion formation in early cardiogenesis: roles of transforming growth factor (TGF)-beta and bone morphogenetic protein (BMP). Anat Rec 258: 119-127
This article was originally published in a special issue, Flexible Mechanism \& Biomechanics handled by Editor. Dr. Qinghua Qin, Australian Nationa University, Australia 